\title{
Probing the Cross-power of Unresolved Cosmic Infrared and X-Ray Backgrounds with Upcoming Space Missions
}

\section{Kashlinsky, A.}

2019-01-20

Kashlinsky , A , Arendt , R G , Cappelluti , N , Finoguenov , A , Hasinger , G, Helgason , K \& Merloni , A 2019 , ' Probing the Cross-power of Unresolved Cosmic Infrared and X-Ray Backgrounds with Upcoming Space Missions ' , Astrophysical Journal Letters , vol. 871 , no. 1,6 . https://doi.org/10.3847/2041-8213/aafaf6

http://hdl.handle.net/10138/298826

https://doi.org/10.3847/2041-8213/aafaf6

cc_by

publishedVersion

Downloaded from Helda, University of Helsinki institutional repository.

This is an electronic reprint of the original article.

This reprint may differ from the original in pagination and typographic detail.

Please cite the original version. 


\title{
Probing the Cross-power of Unresolved Cosmic Infrared and X-Ray Backgrounds with Upcoming Space Missions
}

\author{
A. Kashlinsky ${ }^{1,2}$, R. G. Arendt ${ }^{3,4}$ (D) , N. Cappelluti ${ }^{5}$ (D) , A. Finoguenov ${ }^{6,7}$ (D) , G. Hasinger ${ }^{8}$ (D) , K. Helgason ${ }^{9}$ (D) and A. Merloni $^{10}$ \\ ${ }^{1}$ Code 665, Observational Cosmology Lab, NASA Goddard Space Flight Center, Greenbelt, MD 20771, USA; Alexander.Kashlinsky@nasa.gov \\ ${ }^{2}$ SSAI, Lanham, MD 20770, USA \\ ${ }^{3}$ Code 665, Observational Cosmology Lab, NASA Goddard Space Flight Center, Greenbelt, MD 20771, USA \\ ${ }^{4}$ CRESST II/University of Maryland Baltimore County, Baltimore, MD 21250, USA \\ ${ }^{5}$ Department of Physics, University of Miami, Coral Gables, FL 33124, USA \\ ${ }^{6}$ MPE, Giessenbachstr. 1, Garching D-85748, Germany \\ ${ }^{7}$ Department of Physics, University of Helsinki, Helsinki FI-00014, Finland \\ ${ }^{8}$ European Space Astronomy Centre, E-28691 Villanueva de la Caada, Madrid, Spain \\ ${ }^{9}$ Science Institute, University of Iceland, IS-107 Reykjavik, Iceland \\ ${ }^{10}$ Max-Planck Institute fuer Extraterrestrische Physik (MPE) P.O. Box 1312 Giessenbachstr. 1, D-85741 Garching Germany \\ Received 2018 December 2; revised 2018 December 21; accepted 2018 December 25; published 2019 January 21
}

\begin{abstract}
The source-subtracted cosmic infrared background (CIB) fluctuations uncovered in deep Spitzer data cannot be explained by known galaxy populations and appear strongly coherent with unresolved cosmic X-ray background (CXB). This suggests that the source-subtracted CIB contains emissions from significantly abundant accreting black holes (BHs). We show that theoretically such populations would have the angular power spectrum that is largely independent of the epochs occupied by these sources, provided they are at $z \gtrsim 4$, offering an important test of the origin of the new populations. Using the current measurements we reconstruct the underlying soft X-ray CXB from the new sources and show that its fluctuations, while consistent with a high-z origin, have an amplitude that cannot be reached in direct measurements with the foreseeable X-ray space missions. This necessitates application of the methods developed by the authors to future IR and X-ray data sets, which must cover large areas of the sky in order to measure the signal with high precision. The LIBRAE project within ESA's Euclid mission will probe source-subtracted CIB over one-half of the sky at three near-IR bands, and its cross-power with unresolved CXB can be measured then from the concurrent eROSITA mission covering the same areas of the sky. We discuss the required methodology for this measurement and evaluate its projected signal-to-noise ratio to show the unique potential of this experimental configuration to accurately probe the $\mathrm{CXB}$ from the new $\mathrm{BH}$ sources and help identify their epochs.
\end{abstract}

Key words: cosmology: observations - dark ages, reionization, first stars - diffuse radiation

\section{Introduction}

The near-IR source-subtracted cosmic infrared background (CIB) fluctuations can probe emissions from early stars and black holes (BHs), inaccessible to direct telescopic studies (Cooray et al. 2004; Kashlinsky et al. 2004; Kashlinsky 2005). Analysis of deep Spitzer images, specifically assembled for this measurement (Arendt et al. 2010), revealed source-subtracted CIB fluctuations at 3.6 and $4.5 \mu \mathrm{m}$ (Kashlinsky et al. 2005) significantly exceeding those from the remaining known galaxy populations (Kashlinsky et al. 2005; Helgason et al. 2012) and indicating new cosmological sources. Follow-up studies identified the CIB fluctuation excess to $\sim 1^{\circ}$ with similar levels across the sky (Kashlinsky et al. 2007, 2012; Cooray et al. 2012). The source-subtracted CIB fluctuations at 3.6 and $4.5 \mu \mathrm{m}$ appear coherent with soft $((0.5-2) \mathrm{keV})$ unresolved cosmic X-ray background (CXB; Cappelluti et al. 2013, 2017; MitchellWynne et al. 2016; Li et al. 2018). The coherence levels indicate a much larger proportion of accreting BHs among the new sources than in known populations (Helgason et al. 2014). Two suggestions have been made for the origin of these populations, both at high $z$ : (1) direct collapse BHs (Yue et al. 2013) and (2) LIGO-type primordial BHs making up dark matter (Kashlinsky 2016). See the review by Kashlinsky et al. (2018).

We identify the X-ray auto-power from the new sources and discuss prospects for probing it with the forthcoming eROSITA
$\mathrm{X}$-ray mission in conjunction with the source-subtracted CIB measurements from the Euclid-LIBRAE ${ }^{11}$ project. The reconstructed CXB power from these new sources is so weak that it cannot be isolated directly in the current or forthcoming $\mathrm{X}$-ray missions. The proposed experimental configuration thus appears uniquely suitable in identifying important information about the BH sources responsible for the observed coherence and their epochs and distribution. After specifying the instrumentational configuration (Euclid-LIBRAE for CIB and eROSITA for $\mathrm{CXB}$ ), we define theoretical expectations and show that, unlike low- $z$ sources, BHs at high $z$ exhibit a welldefined shape of the auto- and cross-power spectrum, which rises at $2 \pi / q<0.5$, robustly peaks at $\sim 2^{\circ}-3^{\circ}$, and traces the Harrison-Zeldovich (HZ) regime, $P \propto q$, at larger angular scales $2 \pi / q$. These CXB-CIB cross-powers between sourcesubtracted LIBRAE-based CIB and the net (unclipped) CXB from the X-ray missions are derivable using only harmonics corresponding to their common resolution. We evaluate the uncertainties in this measurement, which require inputs in the net X-ray and IR powers for this setup. The eROSITA-Euclid configuration currently appears to be the most optimal to probe the cross-power and the CXB power arising in these $\mathrm{BH}$ populations.

\footnotetext{
${ }^{11}$ https://www.euclid.caltech.edu/page/Kashlinsky\%20Team
} 


\section{Motivation}

Source-subtracted CIB fluctuations will be measured in three NISP bands from the Euclid Wide Survey (EWS) covering $15,000 \mathrm{deg}^{2}$ to $\mathrm{AB} \sim 25$ with instantaneous $\mathrm{FoV}$ of $0.5 \mathrm{deg}^{2}$ (Laureijs et al. 2011, 2014). Even excluding areas with substantial Galactic foregrounds, EWS will provide sourcesubtracted CIB power to subpercent statistical accuracy. The clipping fraction for CIB maps from Euclid will be $\lesssim 10 \%$ (Kashlinsky et al. 2018) requiring no significant masking corrections of Fourier amplitudes, $\Delta_{1}(\boldsymbol{q})$. Using EWS data, LIBRAE will measure source-subtracted CIB anisotropies on subdegree angular scales allowing to probe the CIB-CXB crosspower with the data from the contemporaneous eROSITA X-ray mission. eROSITA will survey the full sky with $15^{\prime \prime}-28^{\prime \prime}$ resolution after 6 months (eRASS1), and add repeated coverage over the next 3.5 years to reach $\sigma_{\mathrm{X}} \sim 10^{-14} \mathrm{erg} \mathrm{s}^{-1} \mathrm{~cm}^{-2}$ (eRASS8). Additional depth is reached at the polar region of $140 \mathrm{deg}^{2}$ (Merloni et al. 2012). See Figure 1(c).

Cross-power between backgrounds at bands 1 (IR) and 2 (X-ray) is:

$$
P_{12}=\int_{0}^{z_{1}} \frac{\partial F_{1}}{\partial z} \frac{\partial F_{2}}{\partial z} \frac{\mathcal{P}\left(q d_{A}^{-1}(z) ; z\right)}{d_{A}^{2} c H^{-1}(z)} d z
$$

where the Hubble constant is $H(z)=H_{0}\left[\Omega_{\mathrm{m}}(1+z)^{3}+\Omega_{\Lambda}\right]^{1 / 2}$, the comoving angular diameter distance $d_{A}(z)=c \int_{0}^{z} H^{-1}(z) d z$, and $1+z_{1}=\lambda_{1} / \lambda_{\mathrm{Ly} \alpha}=15\left(\lambda_{1} / 1.8 \mu \mathrm{m}\right)$ is the Lyman cutoff for emissions reaching filter at (rest-frame IR) wavelength $\lambda_{1}$, with $\partial F_{1} / \partial z$ being the flux rate production at that wavelength (e.g., NISP $H$-band). For scales subtending linear density field, the 3D power spectrum is linearly biased with respect to the underlying $\Lambda \mathrm{CDM}$ power, $\mathcal{P}(k, z)=b_{1}(z) b_{2}(z) \mathcal{P}_{\Lambda \mathrm{CDM}}(k, z)$. In linear regime and at $z \gtrsim 3$ density perturbations grow so that $(1+z)^{-2} \mathcal{P}_{\mathrm{ACDM}}(k, z)=$ const, which is shown in Figure 1(a) at various $z$. At $z=12$ the comoving distance is $d_{A} \equiv$ $d_{0} \simeq 6.8 h^{-1}$ Gpc varying by $\sim \pm 5 \%$ over $9<z<16$, the range defined by the Ly $\alpha$ cutoff at Euclid/NISP $J, H$ filters. Figure 1(a) shows that sources at $z \gtrsim 5$ exhibit an approximately $z$-independent power spectrum template that robustly defines the cross- and auto-power shapes from sources at those epochs; i.e., the measured cross-power $P_{12} \propto \mathcal{P}_{\Lambda \mathrm{CDM}}\left(q / d_{0}\right) \times$ (the weighted measure of CXB and CIB from these sources). The HZ regime, $\mathcal{P} \propto k$, results in cross- and auto- powers $P \propto q$ and is reached at $2 \pi / q \gtrsim 2^{\circ}-3^{\circ}$. At the same time, sources at low(er) $z$ have widely varying shapes of $\mathcal{P}\left(q / d_{A}\right)$ likely resulting in a broad range of possible shapes for the projected power, which will differ noticeably from the high- $z$ component. Thus measuring the CIB-CXB cross-power with good accuracy over sufficiently wide angular scales is important in probing/verifying the origin of the signal at high $z$.

The CIB-CXB cross-power can be used to assess the CXB fluctuations, $\left[\frac{q^{2}}{2 \pi} P_{\mathrm{CXB}}\right]^{1 / 2}$, from:

$$
S \equiv \delta F_{\mathrm{CXB}}(q)=\sqrt{\frac{q^{2}}{2 \pi} \frac{P_{12}^{2}}{P_{1}}}=\frac{\frac{q^{2}}{2 \pi} P_{12}(q)}{\delta F_{\mathrm{CIB}}(q)} .
$$

This represents the CXB fluctuations produced directly by the new sources to within the product with the square-root of the underlying $\mathrm{CXB}-\mathrm{CIB}$ coherence, currently measured to exceed $\sqrt{\mathcal{C}} \gtrsim 0.4 ;$ if the CIB is predominantly $\mathrm{BH}$-produced Equation (2) would give the true underlying $\mathrm{CXB}$ from the new sources with $\mathcal{C} \simeq 1$. Because coherence is $\leqslant 1$, Equation (2) sets a lower limit on the CXB power from the sources.

From the Spitzer/Chandra-measured cross- and auto-CIB powers $\left(P_{12}^{\mathrm{IRAC}}, P_{\mathrm{CIB}}^{\mathrm{IRAC}}\right)$ one can derive the $\mathrm{CXB}$ power, Equation (2), as $S=\frac{q}{\sqrt{2 \pi}} P_{12}^{\mathrm{IRAC}} /\left[P_{\mathrm{ClB}}^{\lambda_{\mathrm{RRC}}}\right]^{1 / 2}$. Figure 1(b) shows the CXB power reconstructed from the Spitzer-Chandra measurements of Cappelluti et al. (2017). The green line shows the CXB power from the new sources with the normalization of the CIB power using the $\Lambda$ CDM template at $d_{A}=7 h^{-1}$ Gpc normalized per Kashlinsky et al. (2015). While the fit is statistically acceptable, the figure shows the limitations of the current measurements for proper interpretation: the signal is probed with limited accuracy and is restricted to $\lesssim 20^{\prime}$.

Figure 1(c) shows the depth and angular coverage from $\mathrm{X}$-ray data. Figure $1(\mathrm{~d})$ shows $\delta F_{\mathrm{CXB}}(q)$ from sources remaining in the data compared to the signal from populations responsible for the measured source-subtracted CIB fluctuations and cross-power with unresolved CXB. We estimated the angular power spectrum from known extragalactic X-ray point sources following Helgason et al. (2014). AGNs are sparse and bright; therefore, their power spectrum tends to be shot-noise dominated out to $\sim$ a few degrees (Helgason et al. 2014; Kolodzig et al. 2017). We adopt the X-ray population model of Gilli et al. (2007), in agreement with observed source counts (e.g., Luo et al. 2017), and calculate the associated shot-noise power below a given flux limit, neglecting the clustering term from AGN. Galaxies contain high- and low-mass X-ray binaries whose X-ray luminosities scale with star formation rate and stellar mass respectively. For the galaxy population and its clustering, we use a semi-analytic galaxy formation model based on the Millennium simulation (Henriques et al. 2015), which reproduces the observed star formation history and stellar mass function as a function of redshift. We assign each source its luminosity distance and an X-ray brightness using the $L_{X}-\mathrm{SFR} / M_{\star}$ relation from Lehmer et al. (2016), accounting for its scatter. We create a model X-ray image inserting each source in its projected position. Eliminating sources above a given flux limit, the power spectrum is calculated directly from the image using the 2D FFT.

Figure 1(d) shows that to robustly probe the new sources in direct CXB measurements, one would need integrations significantly deeper than what will be available while doing this over a large sky area. Hence, the potential of the proposed CIB-CXB cross-power measurement using Euclid and eROSITA.

The cross-power between source-subtracted CIB from LIBRAE/Euclid and CXB from eROSITA can be evaluated as follows: (1) take X-ray diffuse maps with minimal (corresponding to the X-ray survey limits) clipping and keep all harmonics in $\Delta_{2}(\boldsymbol{q})$, (2) take clipped CIB maps and keep only the same harmonics in $\Delta_{1}(\boldsymbol{q})$. Then (3) evaluate the cross-power over $2 \pi / q>\sigma_{0}$ as $P_{12}(q)=\left\langle\left|\Delta_{1}(\boldsymbol{q}) \Delta_{2}^{*}(\boldsymbol{q})\right| H\left(2 \pi / q-\sigma_{0}\right)\right\rangle$, where the CIB power is (presumably) dominated by the new sources. $\left(H(x)\right.$ is the Heaviside step-function and $\sigma_{0} \sim 30^{\prime \prime}$ for eROSITA). This is similar to the methodology for measuring CMB-CIB cross-power with Euclid all-sky CIB data to probe the 

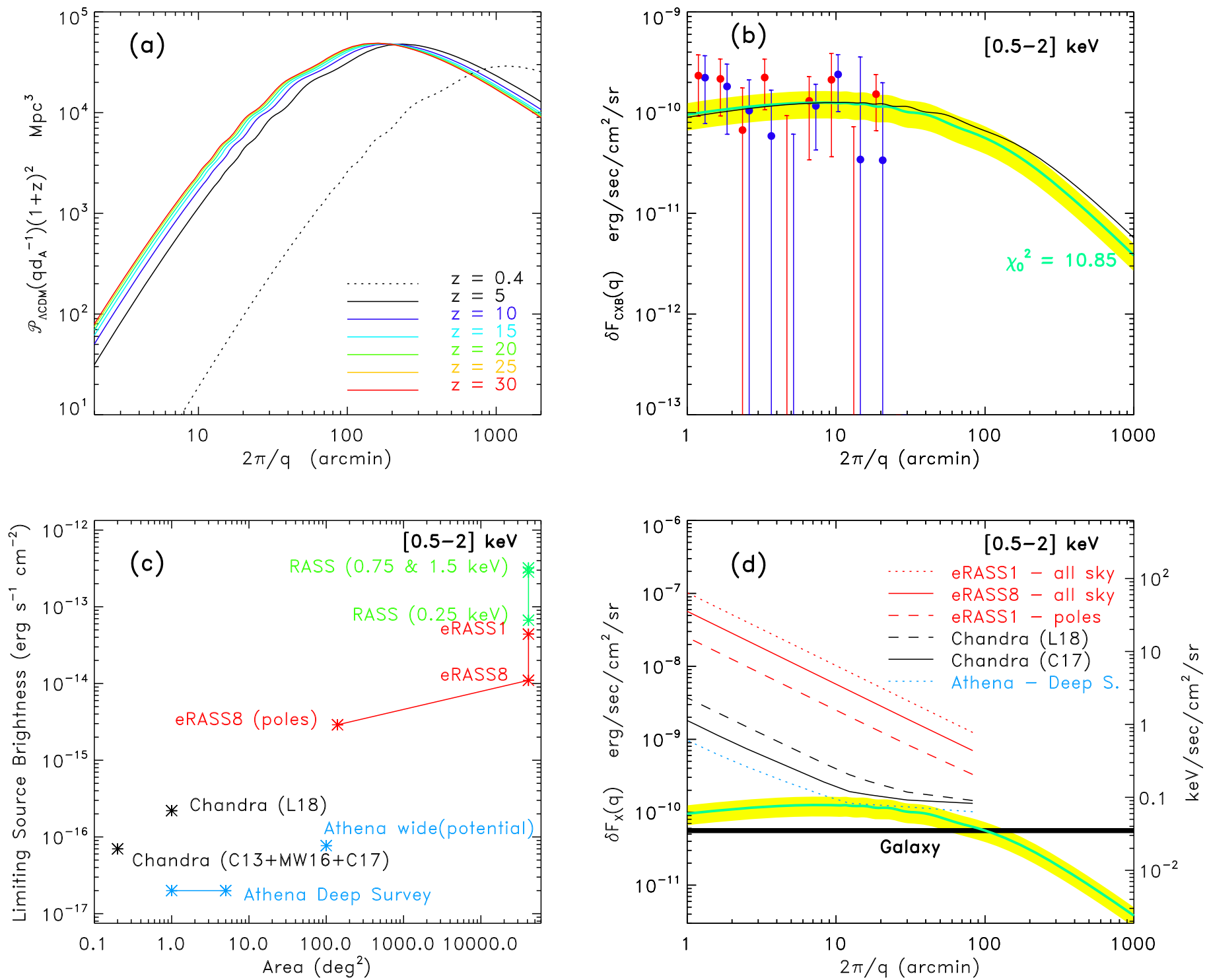

Figure 1. (a) Underlying $\Lambda \mathrm{CDM}$ power spectrum, $\mathcal{P}_{\Lambda \mathrm{CDM}}\left(q / d_{A}\right)$, at $z=0.4,5,10,15,25,30$ projected to their corresponding distances $d_{A}=1.1,5.5,6.6,7.2,7.5$, $7.9 h^{-1} \mathrm{Gpc}$. At $z \gtrsim 3$ the universe is in the Einstein-deSitter regime with density fluctuations growing $\propto(1+z)^{-1}$, so the power is shown multiplied by $(1+z)^{2}$. (b) Blue $(3.6 \mu \mathrm{m})$ and red $(4.5 \mu \mathrm{m})$ circles with errors show $S$ derived using the IRAC/Chandra measurements of Cappelluti et al. (2017). Solid green line is the best fit assuming (1) the power over $1^{\prime}<2 \pi / q<20^{\prime}$ combining the data at 3.6 and $4.5 \mu \mathrm{m}$ and (2) the template from (a) projected to $d_{A}=7 h^{-1} \mathrm{Gpc}(z=15)$; the yellow regions mark the $1 \sigma$ deviation of the fit, which gives $\chi_{0}^{2}=10.85$ for 18 data points (assuming the IRAC bands, with separate optical paths, give independent measurements). Black solid line shows the template projected to $z=5$. (c) X-ray sensitivities. (d) Residual CXB, $\delta F_{X}$, from sources fainter than sensitivity displayed in (c) compared with CXB from the new populations identified with the CIB-CXB coherence. Thick horizontal line shows Galactic X-ray fluctuations (extrapolated from measurements; see Section 3).

IGM at $z \gtrsim 10$ (Atrio-Barandela \& Kashlinsky 2014). The known sources will contribute only negligibly to $P_{12}(q)$ as we discuss in Section 3, but will contribute to the noise on it, Equation (3). The half-energy-width (HEW) of the eROSITA PSF is $28^{\prime \prime}$ with extended tails, so mask leakage from bright sources is a potential noise source. The effects were tentatively discussed in Kashlinsky et al. (2018, Section VII.D) and appear subdominant at small angular scales, becoming less important at larger scales. Conservatively we take the smallest angular scale to be $\sim 1^{\prime}$ for the CXB-CIB cross-power estimates, where also $\mathrm{CIB}$ contributions from the remaining known sources are smaller than the expected high- $z$ component.

In the presence of X-ray and IR maps with net diffuse light power $P_{\mathrm{CIB}}(q), P_{\mathrm{CXB}}(q)$ the error on the measured cross-power at $q$ from an ensemble of $n_{q}$ independent Fourier elements is

$$
\sigma_{\mathrm{IR}, \mathrm{X}}=\sqrt{\frac{P_{\mathrm{IR}} P_{\mathrm{X}}}{2 n_{q}}} .
$$

The cosmological cross-power is expressable in terms of the cosmological auto-powers as $P_{12}(q)=\sqrt{\mathcal{C}(q) P_{\mathrm{CIB}}(q) P_{\mathrm{CXB}}(q)}$ with the coherence potentially reaching $\mathcal{C} \sim 1$. The signal-tonoise of the cross-power measurements, for one single patch of size $\Theta_{\text {patch }}$ on the side, is

$$
\left.(\mathrm{S} / \mathrm{N})\right|_{\mathrm{patch}}=\left[2 n_{q}\right]^{1 / 2}\left[\frac{\delta F_{\mathrm{CXB}}(q)}{\delta F_{\mathrm{X}}(q)}\right]\left[\frac{\delta F_{\mathrm{CIB}}(q)}{\delta F_{\mathrm{IR}}(q)}\right],
$$




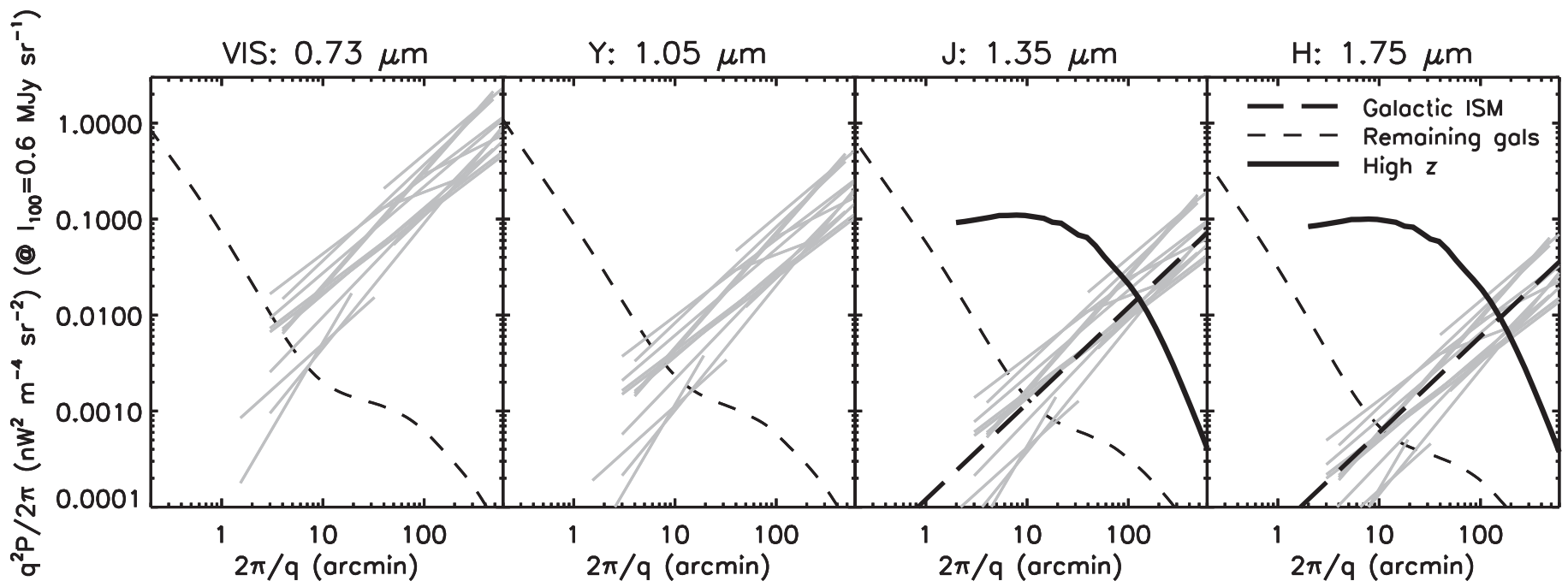

Figure 2. Normalized cirrus power spectra (gray power laws) from various published studies translated to the Euclid wavelengths. Legend marks IR components adopted in subsequent $\mathrm{S} / \mathrm{N}$ estimates: (1) the contribution from remaining known galaxies, (2) the Galactic ISM/cirrus, and (3) the $\Lambda$ CDM power spectrum of the modeled high- $z$ CIB normalized to Spitzer CIB data.

where $\delta F_{\mathrm{X}}=\left[q^{2} P_{\mathrm{X}} /(2 \pi)\right]^{1 / 2}$ is the net diffuse flux fluctuation in the $\mathrm{X}$-ray maps from sources remaining at the $\mathrm{X}$-ray depth and Galaxy, $\delta F_{\mathrm{IR}}$ is defined similarly at its own IR depth. The number of elements, $n_{q}$, for the patch, which goes into determining the power at each $q$ depends on the patch area, $\Theta_{\text {patch }}^{2}$, and the $q$-binning. We write $n_{q} \simeq \pi\left(\frac{\Theta_{\text {path }}}{2 \pi / q}\right)^{2}\left(\frac{\Delta q}{q}\right)$, where $\Delta q$ is the bin-width over which the power at the given wavenumber $q$ is averaged in the Fourier plane. The direct measurement of $\delta F_{\mathrm{CXB}}$ (Equation (2)) would have a corresponding $\left.(\mathrm{S} / \mathrm{N})\right|_{\text {direct }}=\left[n_{q}\right]^{1 / 2}\left[\frac{\delta F_{\mathrm{CX}}(q)}{\delta F_{\mathrm{X}}(q)}\right]^{2}$. Employing the cross-power instead of direct measurement of the CXB fluctuations leads to an improved signal-to-noise ratio $(\mathrm{S} / \mathrm{N})$ when $(2 \mathcal{C})^{1 / 2}\left[\delta F_{\mathrm{CIB}}(q) / \delta F_{\mathrm{IR}}(q)\right]>\delta F_{\mathrm{CXB}}(q) / \delta F_{\mathrm{X}}(q)$. Thus improvements are made when the total background is more strongly influenced by the cosmological component in the IR emission than in the X-ray emission, and as long as a low coherence does not counteract the benefit of using cleaner IR data: the method identifies the new CXB contributing populations if (1) they are strongly coherent with the CIB sources, (2) the CIB power of the new sources is isolated, but (3) their $\mathrm{CXB}$ contributions are drowned in the noise and other X-ray sources. Furthermore, a given S/N Gaussian-distributed crosspower corresponds to higher confidence levels than the $\chi^{2}$-distributed auto-power.

The net signal-to-noise over a wide net area $A$ covering $A / \Theta_{\text {patch }}^{2}$ such patches becomes:

$$
(\mathrm{S} / \mathrm{N})^{2}=\sum_{\text {patches }} 2 n_{q}\left[\frac{\delta F_{\mathrm{CXB}}(q)}{\delta F_{\mathrm{X}}(q)}\right]^{2}\left[\frac{\delta F_{\mathrm{CIB}}(q)}{\delta F_{\mathrm{IR}}(q)}\right]^{2} \equiv 2 n_{q} W(q),
$$

where $W(q) \equiv \sum_{\text {patches }}\left[\frac{\delta F_{\mathrm{CXB}}(q)}{\delta F_{\mathrm{X}}(q)}\right]^{2}\left[\frac{\delta F_{\mathrm{CIB}}(q)}{\delta F_{\mathrm{IR}}(q)}\right]^{2}$ is evaluated in Section 3; this last expression being appropriate when analysis of a large area of sky is performed after dividing it into smaller patches. $W \propto A$ when the terms inside the sum for $W$ are $q$-independent.

\section{CXB-CIB Cross-power Uncertainties}

To evaluate the $\mathrm{S} / \mathrm{N}$ one needs the ratios of the cosmic background to total powers in each of the IR and X-ray bands. For CIB, we adopt a theoretical model based on the IMF500 model from Helgason et al. (2016), which fits the Spitzer excess CIB measurements as discussed in Kashlinsky et al. (2015) with the mean formation efficiency per halo of $f_{\star}=0.04$ ending at $z_{\text {end }}=10$. This model, shown in Figure 2, has negligible CIB contributions in the NISP Y filter, but dominates remaining known galaxy contributions at $J$ and $H$. Strictly speaking it corresponds to stellar emissions from very massive stars $\left(500 M_{\odot}\right.$ each), but, because those radiate at the Eddington limit with $L \propto M$ as do BHs, they can be straightforwardly rescaled to $\mathrm{BH}$ emissions.

In deep IR images, source subtraction removes Galactic stars and resolved extragalactic sources. At small angular scales (e.g., $<20^{\prime \prime}$ ) or for relatively shallow observations, the shot noise of the remaining faint galaxies of known populations will still contribute to the overall IR background power. Emission from the Galactic ISM will also remain, and provide a potentially dominant foreground to the CIB. The power spectrum of Galactic ISM emission has been measured at $100 \mu \mathrm{m}$ (e.g., Gautier et al. 1992; Wright 1998; MivilleDeschênes et al. 2002; Kiss et al. 2003; Lagache et al. 2007; Bracco et al. 2011; Pénin et al. 2012). These studies were in regions of various ISM brightness, but we empirically find using the data from these studies that $P\left(100^{\prime}\right) \propto\left\langle I_{100}\right\rangle^{2.7}$, and renormalize all the reported power spectra to a mean ISM $100 \mu \mathrm{m}$ intensity of $0.6 \mathrm{MJy} \mathrm{sr}^{-1}$. We rescaled the power spectra from $100 \mu \mathrm{m}$ to near-IR using a typical $2-300 \mu \mathrm{m}$ spectrum of the ISM, ${ }^{12}$ extended to shorter wavelengths using the diffuse Galactic light measurements of Brandt \& Draine (2012), and adjusting at $<4 \mu \mathrm{m}$ to match the redder 3.6/ $100 \mu \mathrm{m}$ color (Arendt \& Dwek 2003). The resultant ISM power spectra at different Euclid wavelengths are shown in Figure 2, along with the nominal $P \propto(2 \pi / q)^{3}$ power law that we use to represent the ISM in further calculations, i.e., $P \propto q^{-3}\left\langle I_{100}\right\rangle^{2.7}$.

\footnotetext{
$\overline{12}$ https://irsa.ipac.caltech.edu/data/SPITZER/docs/files/spitzer/ background.pdf
} 

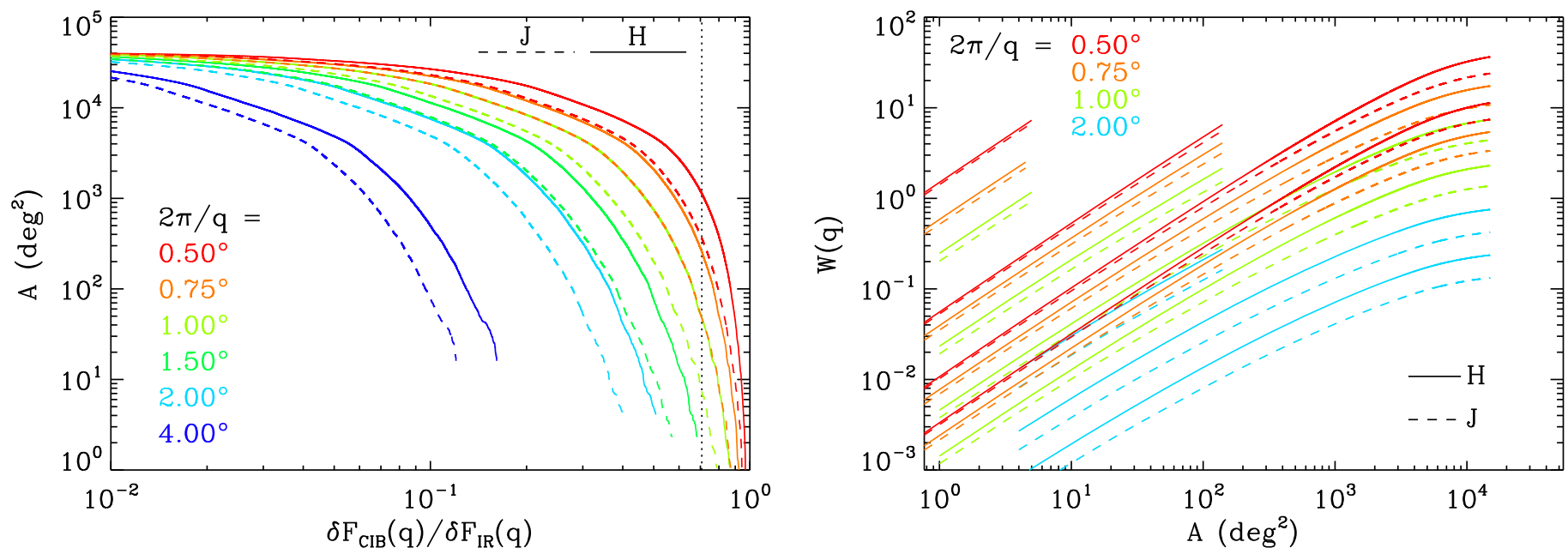

Figure 3. Left: the area over which $\delta F_{\mathrm{CIB}}(q) / \delta F_{\mathrm{IR}}(q)$ exceeds a given value as a function of $2 \pi / q$ and IR band. The expected contributions of emission from the Galactic ISM limits that area of the sky where the CIB can be well measured, especially at large $2 \pi / q$. Dotted vertical line shows $\delta F_{\mathrm{CIB}}(q) / \delta F_{\mathrm{IR}}(q)=\sqrt{0.5}$ identifying where $P_{\mathrm{CIB}}>0.5 P_{\mathrm{IR}}$. Right: $\delta F_{\mathrm{CIB}}(q) / \delta F_{\mathrm{IR}}(q)$ is combined with comparable X-ray evaluations to estimate $W(q) \propto(\mathrm{S} / \mathrm{N})^{2}$ expected for IR-X-ray crosspower measurements made over increasingly larger areas of the sky, $A . W(q) \propto A$ up to $A \sim 10^{2} \mathrm{deg}^{2}$, but as larger areas are affected by higher foregrounds, the $\mathrm{S} / \mathrm{N}$ accumulates more slowly. The curves limited to $A<5$ and $140 \mathrm{deg}^{2}$ represent the Athena-Deep and eRASS poles surveys. The eRASS1 and eRASS 8 surveys cover the entire EWS area, with the deeper eRASS8 providing higher $\mathrm{S} / \mathrm{N}$.

Contributions from remaining known galaxies, dashes in Figure 2 (see Section VII.C, Kashlinsky et al. 2018), are small compared to the model CIB at the scales and wavelengths of interest, $2 \pi / q>1^{\prime}$ and $J, H$. Because of the much deeper IR threshold of removing individual sources in EWS than in eROSITA, they contribute only negligibly to the IR-X-ray cross-power. We evaluated the cross-power from known sources remaining at the EWS $H$-band projected depth using the methodology described in Section 2. The known galaxies were found to contribute $q^{2} P_{12} /(2 \pi) \simeq$ $10^{-12}\left(\mathrm{erg} \mathrm{s}^{-1} \mathrm{~cm}^{-2} \mathrm{sr}^{-1} \mathrm{nW} \mathrm{m}^{-2} \mathrm{sr}^{-1}\right)$ at $2 \pi / q \sim 5^{\prime}$, dropping to $\sim 2 \times 10^{-13}$ at $\sim 30^{\prime}$ while reaching $10^{-11}$ at $2 \pi / q=1^{\prime}$. The CIB-CXB fluctuations are measured at $3.6 \mu \mathrm{m}$ in Cappelluti et al. (2017, their Figure 2) to be $\simeq 10^{-11}$ in these units leading to numbers displayed in Figures 1(b), (d). The $H$-band CIB-CXB cross-power from the new sources would be larger by the square root of the corresponding ratio of the CIB powers at the $H$ to $3.6 \mu \mathrm{m}$ bands or a factor of $\gtrsim 4$ per Figure 2, right, assuming the coherence remains constant. This component is thus neglected in the overall budget of $\mathrm{S} / \mathrm{N}$.

The ratio of the amplitudes of the CXB and the total X-ray fluctuations is estimated from the components shown in Figure 1(d). The total X-ray power is the sum of the CXB, the remaining extragalactic component for any given survey, and a Galactic ISM component. The high latitude X-ray power has been measured by Sliwa et al. (2001) using ROSAT, and over a more limited region using much deeper Chandra data by Kolodzig et al. (2018). The largest scale measurements (at $\sim 2^{\circ}$ ) by Kolodzig et al. (2018) can be extrapolated (using $P_{\mathrm{X}} \propto q^{2}$ or $\delta F_{\mathrm{X}}=$ const) as an upper limit on ISM X-ray emission (see Figure 1(d)), consistent with the largest scale ROSAT/RASS measurements. Comparison with the expected CXB power spectrum reveals that at high latitudes, the ISM power will be comparable to or fainter than the extragalactic power.

The final consideration in evaluating the $\mathrm{S} / \mathrm{N}$ of the prospective measurement involves $n_{q}$ in the region of measurements. The ratios $P_{\mathrm{CIB}} / P_{\mathrm{IR}}$ and $P_{\mathrm{CXB}} / P_{\mathrm{X}}$ discussed above are representative for high latitude regions. For regions closer to the Galactic plane, the foreground emission increases relative to the cosmic background. The ratios and thus the $\mathrm{S} / \mathrm{N}$ for such regions decrease. To estimate this effect, we made maps of the amplitudes of $P_{\mathrm{IR}}$ and $P_{\mathrm{X}}$, by rescaling the DIRBE $100 \mu \mathrm{m}$ and ROSAT R4+R7 maps. Thus the $\mathrm{S} / \mathrm{N}$ can be evaluated by summing over increasingly larger areas (increasing $n_{q}$ and/or the number of patches), but decreasingly smaller ratios of background to total power. The resultant $W^{\prime}$ s are shown in Figure 3, right. As one approaches large areas, the value of $\sqrt{W}$, governing the overall achievable $\mathrm{S} / \mathrm{N}$, saturates and increasing the net area leads to progressively lower benefits as $A \gtrsim$ a few thousand $\operatorname{deg}^{2}$.

\section{Measurement Prospects with Upcoming X-Ray Missions}

We now evaluate the $\mathrm{S} / \mathrm{N}$ of the aforementioned configurations using the EWS CIB. The dotted line in Figure 3 (left) demarcates where $\mathrm{CIB}$ and foreground contributions to the net IR power become comparable: $P_{\mathrm{CIB}}$ becomes dominant over the IGM/cirrus contributions over (1) $A \sim 500 \mathrm{deg}^{2}$ area if square patches of 0.7 on the side are selected (comparable to NISP's FoV), (2) $A \sim 100 \mathrm{deg}^{2}$ if the area $A$ is probed in square patches of $1 \mathrm{deg}^{2}$, or $2 \pi / q \lesssim 1^{\circ}$, and (3) $A \sim 2.5 \mathrm{deg}^{2}$ if probing out to $2 \pi /$ $q \sim 1$. 5 . These configurations correspond to (1) eROSITA1/8, (2) eROSITA-poles, and (3) Athena-deep and we estimate the resultant $\mathrm{S} / \mathrm{N}$ there. This is a conservative limit, as covering more area would result in a better signal out to $\mathrm{S} / \mathrm{N} \simeq$ $\sqrt{2 n_{q} \times \max [W(q)]}$; Figure 3 (right) shows that $\gtrsim 50 \%$ of $\mathrm{S} / \mathrm{N}$ for eRASS all-sky configurations is reached at $A \lesssim$ $1000 \mathrm{deg}^{2}$. In estimating $\mathrm{S} / \mathrm{N}$ we adopted Fourier binning of $\Delta q / q=0.1$ and assumed that the patches analyzed are of the same extent as the scale of interest. If the patches analyzed are much larger than the scales measured, these results can be binned over wider $\Delta q$, which increases $n_{q} \propto \Delta q$ and $\mathrm{S} / \mathrm{N} \propto \sqrt{\Delta q}$.

Figure 4 (top) shows the achievable $\mathrm{S} / \mathrm{N}$ for the NISP $H$-band. As Figure 2 indicates, the numbers using $J$-band CIB would be similar; though, combining the two IR bands would not lead to appreciable increase of the overall $\mathrm{S} / \mathrm{N}$ because the uncertainties are due to populations remaining in the data, which correlate between the IR bands, rather than instrumental noise. eROSITA appears to be very well suited for this measurement at least 


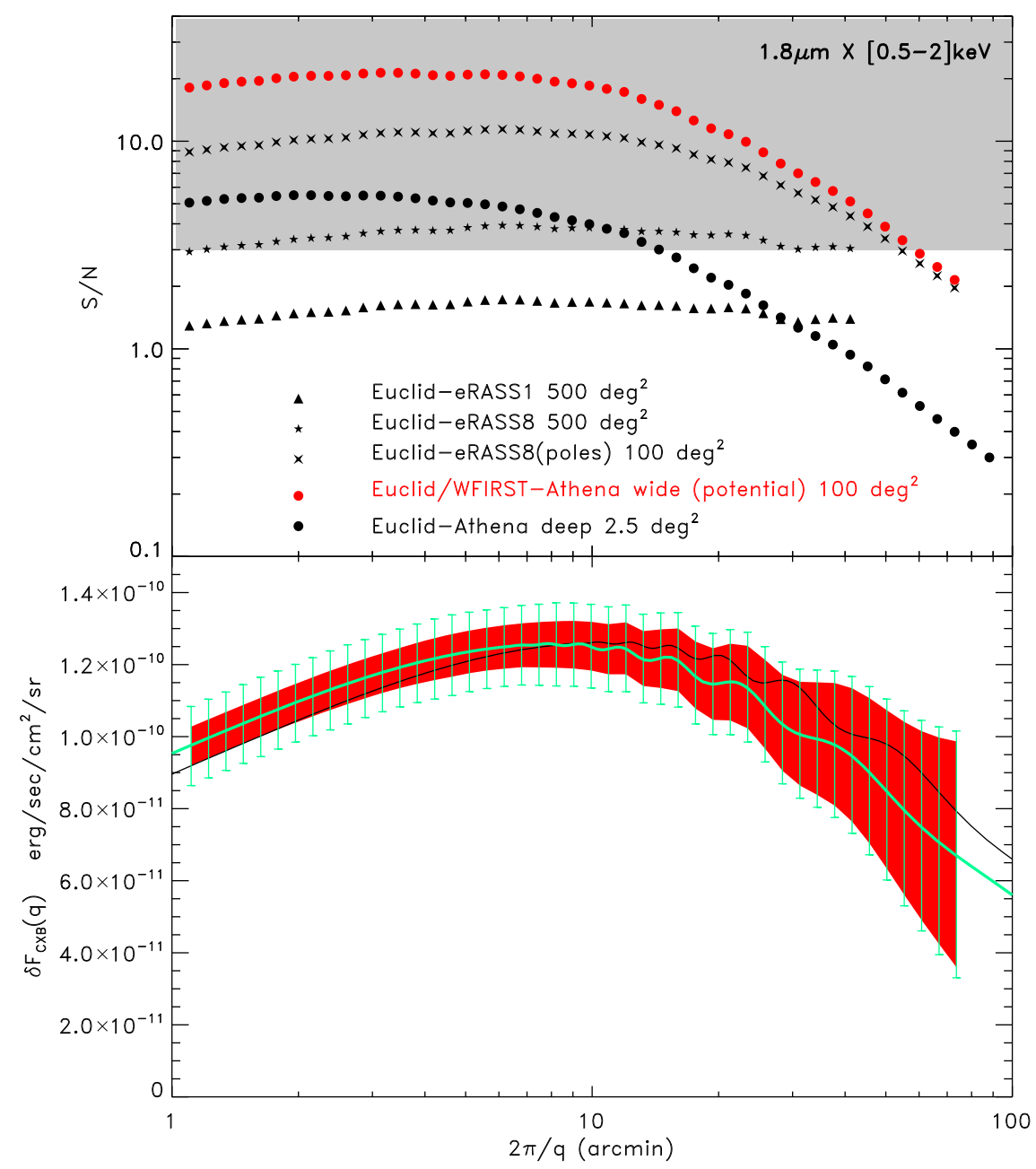

Figure 4. Top: overall $\mathrm{S} / \mathrm{N}$ with $\Delta q / q=0.1$ for NISP's $H$-band CIB for the marked configurations; for $J$-band the numbers would be similar and are not shown for brevity. Shaded area marks $\mathrm{S} / \mathrm{N} \geqslant 3$. Bottom: the expected signal in Figure 1(d) if generated by sources at $z=15$ (green solid) and $z=5$ (black dotted); in reality the cross-power is a weighted sum/integral over $z$. Vertical bars correspond to $1 \sigma$ uncertainties projected in the $e$ ROSITA Poles survey covering $A=100$ deg $^{2}$ and $\Delta q / q=0.1$. Red shows the $1 \sigma$ limits for the same configuration with a potential Athena survey.

as far as the soft X-ray band is concerned, with the best configuration being the eROSITA-poles survey. At a given X-ray depth $\mathrm{S} / \mathrm{N} \propto A^{1 / 2}(\Delta q / q)^{1 / 2} . \mathrm{S} / \mathrm{N} \geqslant 3$ at each scale can be achieved already with the eROSITA1 maps with $\Delta q / q \simeq$ $0.25\left(A / 1000 \mathrm{deg}^{2}\right)^{-1}$ out to $\sim 1^{\circ}$, enabling a good first look at the cross-power. Athena-Deep would have good sensitivity out to $\sim 30^{\prime}$, but because of its small area will lose sensitivity at larger scales. eRASS8 and $e$ ROSITA-poles surveys will allow probing the cross-power highly accurately out to $\sim 2^{\circ}$. We note that, given the high $\mathrm{S} / \mathrm{N}$, masking corrections may be important to make when doing FFTs, unless avoided by the CPU-time intensive correlation function analysis.

The shape of the cross-power, as probed by the eROSITApoles, could potentially provide an indicator of the epochs of the new sources although detailed studies of the masking effects at such high $\mathrm{S} / \mathrm{N}$ would be required to answer this quantitatively as we are now conducting. However, the high- $z$ origin of the signal would be probed directly with the Lymanbreak cutoff from the EWS VIS and NISP cross-powers (Kashlinsky et al. 2018).

Post-Euclid/eROSITA, WFIRST to-be-launched in late 2020 's will cover $\sim 2000 \mathrm{deg}^{2}$ at four NIR bands to deeper levels than EWS (Spergel et al. 2015) and ESA's Athena X-ray mission launch (Nandra et al. 2013) is planned in the early 2030s. Athena with $\sim 5^{\prime \prime}$ HEW PSF and a collecting area much larger than eROSITA's, will be a much more sensitive telescope for faint diffuse emission to study the fluctuations over a broader range of angular scales. An Athena wide survey (say, $A=100 \mathrm{deg}^{2}$ ) at depths comparable to the Chandra integrations used in Cappelluti et al. (2017) will achieve the $\mathrm{S} / \mathrm{N}$ shown with red in Figure 4. This exceeds eROSITA and would require only as much integration as the planned Athena Deep Survey $\left(A \simeq 2-5 \mathrm{deg}^{2}\right)$, assuming $A \sigma_{\mathrm{X}}^{2}=$ const. Such an Athena wide survey would probe the CIB-CXB cross-power, and the corresponding CIB auto-power, at the levels where remaining CXB comes very close to that from the new sources (Figure 1(d)). In this situation one would be able to probe the intrinsic coherence due to BHs among the CIB sources.

We acknowledge support from NASA/12-EUCLID11-0003 "LIBRAE: Looking at Infrared Background Radiation Anisotropies with Euclid" project (http://librae.ssaihq.com), NASA award \#80GSFC17M0002 (R.G.A.), and Icelandic Research Fund grant \#173728-051 (K.H.).

\section{ORCID iDs}

R. G. Arendt (10 https://orcid.org/0000-0001-8403-8548

N. Cappelluti (i) https://orcid.org/0000-0002-1697-186X 
A. Finoguenov (i) https://orcid.org/0000-0002-4606-5403

G. Hasinger (10) https://orcid.org/0000-0002-0797-0646

K. Helgason (1) https://orcid.org/0000-0002-4326-9144

\section{References}

Arendt, R. G., \& Dwek, E. 2003, ApJ, 585, 305

Arendt, R. G., Kashlinsky, A., Moseley, S. H., \& Mather, J. 2010, ApJS, 186,10

Atrio-Barandela, F., \& Kashlinksy, A. 2014, ApJL, 797, L26

Bracco, A., Cooray, A., Veneziani, M., et al. 2011, MNRAS, 412, 1151

Brandt, T. D., \& Draine, B. T. 2012, ApJ, 744, 129

Cappelluti, N., Arendt, R., Kashlinsky, A., et al. 2017, ApJL, 847, L11

Cappelluti, N., Kashlinsky, A., Arendt, R. G., et al. 2013, ApJ, 769, 68

Cooray, A., Bock, J. J., Keatin, B., Lange, A. E., \& Matsumoto, T. 2004, ApJ, 606, 611

Cooray, A., Smidt, J., de Bernardis, F., et al. 2012, Natur, 490, 514

Gautier, T. N. I., Boulanger, F., Perault, M., \& Puget, J. L. 1992, AJ, 103, 1313

Gilli, R., Comastri, A., \& Hasinger, G. 2007, A\&A, 463, 79

Helgason, K., Cappelluti, N., Hasinger, G., Kashlinsky, A., \& Ricotti, M. 2014, ApJ, 785, 38

Helgason, K., Ricotti, M., \& Kashlinsky, A. 2012, ApJ, 752, 113

Helgason, K., Ricotti, M., Kashlinsky, A., \& Bromm, V. 2016, MNRAS, 455,282

Henriques, B. M. B., White, S. D. M., Thomas, P. A., et al. 2015, MNRAS, 451, 2663

Kashlinsky, A. 2005, PhR, 409, 361

Kashlinsky, A. 2016, ApJL, 823, L25

Kashlinsky, A., Arendt, R., Gardner, J. P., Mather, J. C., \& Moseley, S. H. 2004, ApJ, 608, 1
Kashlinsky, A., Arendt, R. G., Ashby, M. L. N., et al. 2012, ApJ, 753, 63

Kashlinsky, A., Arendt, R. G., Atrio-Barandela, F., et al. 2018, RvMP, 90 025006

Kashlinsky, A., Arendt, R. G., Atrio-Barandela, F., \& Helgason, K. 2015, ApJL, 813, L12

Kashlinsky, A., Arendt, R. G., Mather, J., \& Moseley, S. H. 2005, Natur, 438,45

Kashlinsky, A., Arendt, R. G., Mather, J., \& Moseley, S. H. 2007, ApJL, 654, L5

Kiss, C., Ábrahám, P., Klaas, U., et al. 2003, A\&A, 399, 177

Kolodzig, A., Gilfanov, M., Hütsi, G., \& Sunyaev, R. 2017, MNRAS, 466, 3035

Kolodzig, A., Gilfanov, M., Hütsi, G., \& Sunyaev, R. 2018, MNRAS, 473, 4653

Lagache, G., Bavouzet, N., Fernandez-Conde, N., et al. 2007, ApJL, 665 L89

Laureijs, R., Amiaux, J., Arduini, S., et al. 2011, arXiv:1110.3193

Laureijs, R., Racca, G., Stagnaro, L., et al. 2014, Proc. SPIE, 9143, 91430H

Lehmer, B. D., Basu-Zych, A. R., Mineo, S., et al. 2016, ApJ, 825, 7

Li, Y., Cappelluti, N., Arendt, R. G., et al. 2018, ApJ, 864, 141

Luo, B., Brandt, W. N., Xue, Y. Q., et al. 2017, ApJS, 228, 2

Merloni, A., Predehl, P., Becker, W., et al. 2012, arXiv:1209.3114

Mitchell-Wynne, K., Cooray, A., Xue, Y., et al. 2016, ApJ, 832, 104

Miville-Deschênes, M. A., Lagache, G., \& Puget, J. L. 2002, A\&A, 393, 749

Nandra, K., Barret, D., Barcons, X., et al. 2013, arXiv:1306.2307

Pénin, A., Lagache, G., Noriega-Crespo, A., et al. 2012, A\&A, 543, A123

Śliwa, W., Soltan, A. M., \& Freyberg, M. J. 2001, A\&A, 380, 397

Spergel, D., Gehrels, N., Baltay, C., et al. 2015, arXiv:1503.03757

Wright, E. L. 1998, ApJ, 496, 1

Yue, B., Ferrara, A., Salvaterra, R., Xu, Y., \& Chen, X. 2013, MNRAS, 433,1556 